themselves. Aversive stimuli may not cause response suppression if they are delivered contingent on responses which the S considers "correct," and neutral stimuli may cause such suppression if they are delivered contingent on responses which the $S$ considers "incorrect." The results of this study illustrate once again that the effects of response-contingent stimuli upon behavior cannot be predicted without knowledge of the S's previous experiences with those stimuli.

\section{REFERENCES}

AYLlON, T., AZRIN, N. H. Punishment as a discriminative stimulus and conditioned reinforcer with humans. Journal of the Experimental Analysis of Behavior, 1966. 9, 411-420.

AZRIN, N. H. Some effects of noise on human behavior. Joumal of the Experimental Analysis of Behavior, 1958 . $1,183-200$.

AZRIN, N. H. Sequential effects of punishment. Science, 1960, 131, 605-606.
FLANAGAN, B., GOLDIAMOND, 1., \& AZRIN, N. H. Operant stuttering: The control of stuttering behavior through response-contingent consequences. Journal of the Experimental Analysis of Behavior. 1958. 1, 173-178

HOLZ, W. C., \& AZRIN. N. H. Discriminative properties of punishment. Journal of the Experimental Analysis of Behavior, 1961, 4, 225-32.

HOLZ, W. C. \& AZRIN, N.H. Interactions between the discriminative and aversive properties of punishment. Journal of the Experimental Analysis of Behavior, 1962 . 5, 229-234.

HOLZ, W. C., AZRIN, N. H. \& AYLLON T. Elimination of behavior of mental patients by response-produced extinction. Journal of the Experimental Analysis of Behavior, 1963, 6, 407-414.

SIEGEL, G. M.. \& MARTIN, R. R. Punishment of dysfluencies in normal speakers. Journal of Speech \& Hearing Research, 1966, 9, 208-218.

SOLOMON, R. L. Punishment. American Psychologist, 1964, 19, 239-53.

TOLMAN, E. C., HALL, C. S., \& BRETNALL, E. P. A disproof of the Law of Effect and a substitution of the law of emphasis, motivation, and disruption. Journal of Experimental Psychology, $1932,15,601-614$.

\title{
Effects of excessive temperature change on contrast in temperature perçeption
}

\author{
EDWIN A. RUGG and JAMES M. MacDOUGALL \\ Florida Presbyterian College, St. Petersburg, Fla. 33733
}

Contrast in temperature perception was investigated in two experiments to follow up a previous report of perceptual assimilation. The results suggest that perceptual assimilation does not occur; instead, large temperature shifts result in periods of tingling or numbness which appear to mask thermal sensations.

If a $S$ adapts his left hand in a water bath maintained at $25^{\circ} \mathrm{C}$ and his right hand in a bath at $30^{\circ} \mathrm{C}$, and then places both hands in a bath at $33^{\circ} \mathrm{C}$, he will typically report that the left hand feels warmer than the right. This operation defines thermal contrast. A classical interpretation of this result is that during the interval of exposure to the adapting stimuli $\left(25^{\circ}\right.$ and $30^{\circ} \mathrm{C}$, respectively), the warmth threshold shifts to a lower intracutaneous temperature level in the left hand than in the right. Thus, upon transfer to the $33^{\circ} \mathrm{C}$ bath, the temperature gradient for the left hand is steeper than that of the right, and greater subjective warmth results.

A recent study which questions the simplicity of the contrast phenomenon has reported findings of the reversal of classical contrast (i.e., assimilation) in temperature perception (Egeth, Kamlet, \& Bell, 1970). In the paradigm described above, assimilation would occur if the $S$ reported that the right hand felt warmer than the left. Although Egeth et al offer no explanation for the assimilation phenomenon, they note that the occurrence of a contrast or assimilation response may depend on the overall range of temperatures spanned. In their study, each $\mathbf{S}$ reported a single comparative response between the two differently adapted hands immediately following exposure to the test stimulus. Subjective reports describing the independent sensations and the duration of the assimilation phenomenon were not made. It was the purpose of the present experiment to investigate these aspects of the phenomenon. Based upon our results, we would suggest that thermal receptors exposed to excessive temperature shifts may respond typically in accordance with the contrast phenomenon but that the initial response of such receptors may be masked by the excitation of nonthermal receptors caused by extreme stimulation.

\section{EXPERIMENT 1} Method

Four female undergraduates from an introductory course in psychology at Florida Presbyterian College volunteered to participate in the experiment for academic credit. The stimuli were two aluminum plates. One plate, which served as the adaptation stimulus, was kept at a constant temperature of $42^{\circ} \mathrm{C}$ through the use of a constant-temperature water bath. The temperature of the other plate was regulated by a Komatsu thermoelectric freezing unit, and it served as the test stimulus. By holding this plate at $30^{\circ}, 27^{\circ}, 24^{\circ}$, $21^{\circ}$, and $18^{\circ} \mathrm{C}$, five temperature ranges of $12^{\circ}, 15^{\circ}, 18^{\circ}, 21^{\circ}$, and $24^{\circ} \mathrm{C}$ were established between the two plates. Both plates were arranged horizontally in front of the $S$.

The Ss participated individually in 1 - $h$ sessions and completed the procedures twice in separate sessions on different days. One session consisted of four tests at each temperature range. The five temperature-range conditions were presented in the same random sequence to all Ss following a short practice period. The basic procedure involved adapting the volar region between the fingertip and the last joint on a right-hand finger for $90 \mathrm{sec}$ and then placing it and the corresponding unadapted finger of the left hand on the test plate. Ss then gave a descriptive and comparative report of the temperature sensations perceived in the two fingers. The test trial lasted until the $S$ reported subjective equality of both fingers or until $10 \mathrm{sec}$ had elapsed, whichever came first. In each range condition, four different fingers were adapted, thus allowing a 10-min recovery period for each finger between tests. If the $S$ did not discriminate any difference in temperature between the two fingers during the entire test trial, that trial was repeated. Preliminary instructions emphasized the importance of accurate discrimination in each unique trial.

\section{Results and Discussion}

Responses were pooled for all Ss for each range condition. Three response measures were examined: (1) percentage of initial contrast reports, determined from the first discriminated difference between fingers; ( 2 ) percentage of discrimination reversal, indicated by reported reversal of the initial discrimination, and (3) percentage of final contrast reports, determined from the status of the discriminated difference prior to the end of the test period. Scores for these measures are presented as a function of temperature range in Fig. 1. Individual percentage scores for each $\mathbf{S}$ for each measure were then subjected to Friedman's nonparametric rank test. The hypothesis that the effects of temperature range were zero was 


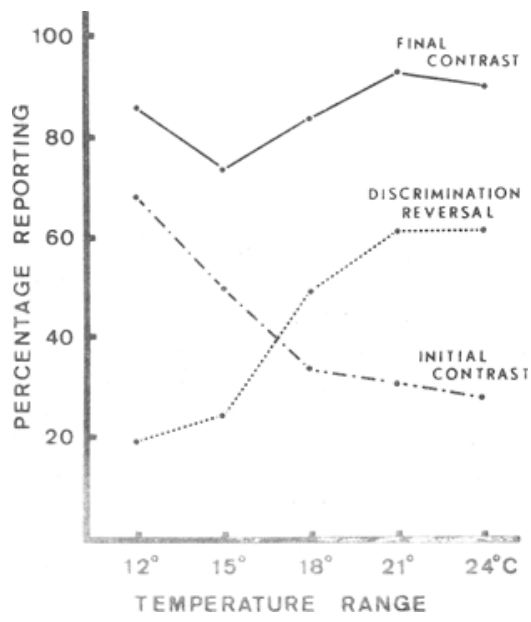

Fig. 1. Percentage scores for measures of initial contrast discrimination reversal, and final contrast as a function of the range of temperature shift.

rejected for initial contrast $(W=.58$, $\mathrm{n}=5, \mathrm{~m}=4, \mathrm{p}<.05)$ and discrimination reversal $(W=.73, n=5$, $\mathbf{m}=4, p<.01$ ), but not for final contrast $(\mathrm{W}=.35, \mathrm{n}=5, \mathrm{~m}=4$, $\mathrm{p}<.2$ ).

The hypothesis suggested by Egeth et al $(1970)$, that occurrence of contrast in the immediate comparative response decreases as a function of increasing temperature range, appears to be supported. It is clear, however, that this effect is short lived, and that the final report is typically that of contrast. Furthermore, Ss usually reported sensations of numbness, prickling, and/or tingling of the adapted finger after the extreme shifts in temperature of the $18^{\circ}, 21^{\circ}$, and $24^{\circ} \mathrm{C}$ ranges, but such reports were rarely made about either finger in the shortest range. In addition, prior to a reversal, Ss generally reported difficulty in defining or detecting thermal sensations in the right finger. This appears to be an unexpected result in light of Zotterman's subhuman findings (1959), which show that cold fibers react to rapid cooling with exaggerated excitation and short latency while warm fibers manifest an off-effect. Such information leads us to suggest that the tingling sensations involved in the longer range reports may represent an interference or masking effect resulting from nonthermal receptor excitation. Furthermore, perhaps interfering sensory input was masking temperature perception to the point that an immediate discrimination may have resulted, not from a comparison of similar stimulus values, but from detection vs nondetection of the stimulus. Experiment 2 was devised to investigate this possibility.

\section{EXPERIMENT 2} Method

Three of the four female participants in Experiment 1 served as ss for this experiment. Constant-temperature water baths were chosen as stimuli instead of plates to eliminate possible confounding effects of applied finger pressure on time required to detect the stimulus. As a result of this choice, apparatus limitations prevented investigation of those ranges used in Experiment 1. Instead, ranges of $7^{\circ}$, $10^{\circ}, 13^{\circ}, 16^{\circ}$, and $19^{\circ} \mathrm{C}$ were created by holding the test stimulus at $24^{\circ} \mathrm{C}$ and the adaptation stimulus bath at $31^{\circ}, 34^{\circ}, 37^{\circ}, 40^{\circ}$, and $43^{\circ} \mathrm{C}$.

The $\mathrm{Ss}$ again participated individually in 1-h sessions and completed the procedures twice in two separate sessions. In each of the five temperature conditions, the $S$ submerged one of four right-hand fingers in the adaptation stimulus for 90 sec. Fingers were submerged to the last joint. The finger was then transferred to the test stimulus, and the $\mathrm{E}$ began recording the elapsed exposure time, using a stopwatch. The $\mathrm{S}$ signaled the $\mathrm{E}$ to stop the watch, using the word "now," as soon as she perceived a distinct cold sensation. Ss were then asked to give descriptive reports of the sensation. All four fingers were tested at each stimulus range on both sessions, with the same random order used for each $\mathrm{S}$.

Results and Discussion

Mean elapsed time for the detection of a distinct cold sensation was determined for each range condition. Figure 2 presents these mean latencies for each of the three Ss. Using Friedman's test, the hypothesis that temperature range had no effect on latency was rejected $(W=.78, n=5$, $m=3, p<.05$ ). In addition, subjective reports again proved interesting. Often in the shorter ranges cold sensations were reported to be relatively instantaneous and approached maximum much more quickly than those in the longer ranges. Furthermore, longer range cold sensations were described as delayed and were often preceded and accompanied by tingling and/or numbness.

These findings show that latencies for the perception of temperature tend

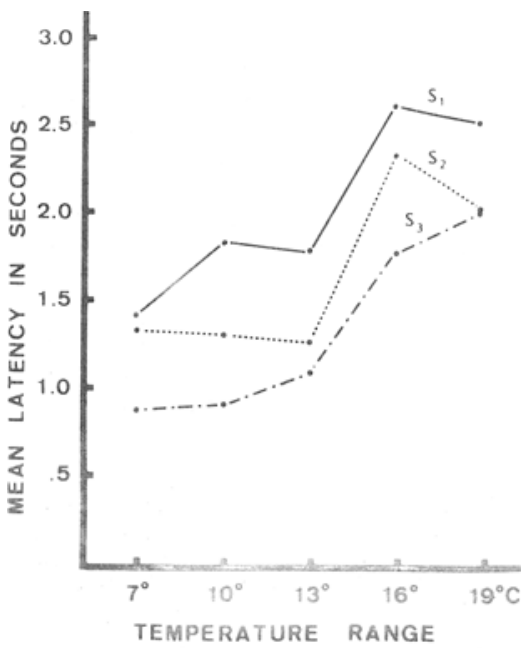

Fig. 2. Mean elapsed time to detection of a distinct cold sensation by the three Ss as a function of the range of temperature shift.

to increase as a function of range of temperature change. Thus, the hypothesis that the initial discriminated difference in Experiment 1 resulted from detection rather than comparison, as described above, is supported. That latencies in the longer ranges may be affected by interference effects is again suggested by the data.

\section{CONCLUSIONS}

Excessive change in temperature was shown to elicit a temporary response tendency resembling assimilation. We feel, however, that this tendency results from comparisons of thermal and nonthermal sensations rather than a true assimilation response. Contrast occurrence was obtained consistently following what appeared to be an initial masking or interference effect from extreme stimulation. Such masking effects are consistent with reports by Zotterman (1959), who attributes such accompanying cutaneous sensations as tingling to interference from some unmyelinated, less specific cutaneous fibers activated by strong abnormal stimuli. The authors are hopeful that this data will facilitate further investigations in this area.

\section{REFERENCES}

EGETH, H. E., KAMLET, A. S., \& BELL, R. A. The reversal of classical contrast in temperature perception. Psychonomic Science, 1970, $19,96$.

ZOTTERMAN, Y. Thermal sensations. In J. Field, H. W. Mogoun, and V. E. Hall (Eds.), Handbook of physiology: Neurophysiology. Vol. 1. Washington: American Physiological Society, 1959. 